\title{
Preliminary analysis of a new ergonomic system for surgical instruments used in laparoscopy
}

\author{
Arturo Minor Martínez ${ }^{1}$, Ricardo Ordorica Flores ${ }^{2}$, Alfredo Vázquez Huerta ${ }^{1}$, \\ José Luis Ortiz Simón ${ }^{3}$ \\ ${ }^{1}$ Department of Electrical Engineering, CINVESTAV del IPN, Mexico, DF \\ ${ }^{2}$ Department of Pediatrics, Children Hospital Federico Gomez, Mexico, DF \\ ${ }^{3}$ Department of Electrical-Electronics Engineering, Instituto Tecnologico de Nuevo Laredo, Tamaulipas, Mexico
}

\section{Email address:}

aminor@cinvestav.mx(A. M. Martínez), ricaordor@hotmail.com(R. O. Flores), avazquezh@cinvestav.mx(A. V. Huerta), jlortizs@itnuevolaredo.edu.mx(J. L. Ortiz Simón)

\section{To cite this article:}

Arturo Minor Martínez, Ricardo Ordorica Flores, Alfredo Vázquez Huerta, José Luis Ortiz Simón. Preliminary Analysis of a New Ergonomic System for Surgical Instruments Used in Laparoscopy. Journal of Surgery Vol. 1, No. 2, 2013, pp. 28-31.

doi: $10.11648 /$ j.js.20130102.15

\begin{abstract}
Background. The laparoscopic surgeon requires very fine motor dexterity to perform complex surgical procedures. This skill can be significantly altered by the motor stress of the surgeon, which mainly results from a lack of ergonomics of the instruments. The objective of this article was to analyse a new ergonomic system that can significantly reduce motor stress. Material and Methods. Two expert surgeons tested the new ergonomic system by performing transfer and suture tasks. The results were compared to those obtained from executing the same tasks using commercial laparoscopic instruments, without any pre-selection being established. Electronic switches were placed on the handles of the instruments to register the time of opening and closure, as well as the time taken to complete the tasks. Results. The comparative data indicate that the new ergonomic system substantially reduces the motor stress of the surgeon by reducing the number of manual operations needed to carry out the routine training tasks of transfer and suture. Conclusions. A new ergonomic system has been developed that reduces the number of manual operations required for carrying out the same task using commercial laparoscopic instruments. The execution time and motor stress is substantially decreased with this new design. We have confidence that these results will be extended to surgical procedures.
\end{abstract}

Keywords: Instrumental Ergonomics, Motor Stress, Ergonomic Optimization

\section{Introduction}

Laparoscopic surgery is considered to be vanguard surgery par excellence. This technique allows for surgical procedures to be performed through access ports of very small diameter. Gas, cold light, visual recording systems, and specialised surgical instruments are introduced into the body through these ports. The gas and light provide adequate work space and lighting, respectively, of the organs and tissues. The surgical instruments can reach their targets and the surgeon can continue with the cutting, dissecting, suturing, or stapling tasks. The instruments used nowadays are different lengths and have diverse handles, with different designs, allowing the surgeon to hold and manipulate them. The surgeon needs ergonomic surgical equipment due to the diversity of the movements performed during surgery. It has been reported that, due to the great diversity of movements and postures required to reach a specific surgical target, the surgeon may suffer articular and tendon injuries, such digital nerve compression, which can make future surgical work impossible [1]. These injuries happened because the surgeon is focused on the surgical target only, not the position of his arms and hands or the force exerted by the fingers while he is trying to reach the target. Different authors have addressed the problem of the lack of ergonomics with different proposals [2-7]. However, we believe there is a key factor that is crucial to help surgeons: the number of openings and closures used during normal use of surgical instruments. If it were possible to reduce this number, the effort exerted (physical energy and muscle stress) by the surgeon would be substantially reduced. In this article, we propose and analyses an ergonomic handle with a mechanical system that keeps the instrument tip closed under normal 
conditions. This report analyses the performance of the ergonomic system during transfer and suture tasks.

\section{Material and Methods}

Two tasks were selected to evaluate the new ergonomic system. The transfer task was chosen because it requires coordination between both hands and visuospatial adaptation. Additionally, there is a lot of statistical information available about the timing to complete this task. The task of suture was also selected, since it requires great surgical skills to execute. In addition to coordination and visuospatial adaptation, it requires refined motor skills and performance in order to handle the needle. Additionally, movements are more specialised for easier suturing. Two forceps were chosen for the adaptation of the new ergonomic handle. The first is a grasper forceps and the second is a needle driver. The handle was coupled to the mechanical system (Fig. 1). The semi-spherical handle, which in conjunction with the grasper, allows one to support the palm of the hand and fingers while opening of the instrument. A spring systems is compressed to maintain the tip of the instrument normally closed. The compression of the spring increases or reduces the pressure of the grasper tip. It can be adjusted manually.

Two expert surgeons tested the instruments. First, they used commercial surgical instruments (five times for each task, 10 in total). There was no pre-selection in the handle. Afterwards, they carried out the same tasks (five times for each task, 10 in total) using instruments that were modified with the new system. All of the tests (20 in total) were carried out in a physical trainer with a mini colour camera serving as a viewpoint at 0 degrees. A commercial 20 inch television screen was used as a monitor. Electro-optic switches were placed in the handles of the instruments to measure the time for opening and closure of the handles of the instruments (Fig. 2a and 2b). The partial time of openings and closures was registered by optical sensors located on the handle and analysed by a computer. The total time to complete the task was also registered.

\subsection{Transfer Task Description}

Transfer task is the classic assignment where rubber rings, arranged in lines on a board, are lifted with the left hand and transferred to the right hand. Then, the rubber ring is placed on right side of the board. This operation is then reversed and the task ends when the rubber rings are placed back to the original position.

\subsubsection{Transfer Task Using Conventional Instrument}

When using traditional surgical instruments, the surgeon prepared the capture of the ring by opening the forceps tip; he visually located on the monitor the ring that was to be moved. He captured the ring and holds it, applying pressure on the handle to keep it closed, thus preventing the ring from falling down. With the other hand, the surgeon opens the receptor forceps and, again using the monitor as visual guide, passed the ring from one hand to the other. The surgeon held the ring and moved it to the final destination, while applying pressure to the handle, and transferred the ring by dropping it in its place. Table 1 shows the timing scores to complete the task using the conventional instruments. Fig. 3 shows the number of openings and closures of forceps during the third repetition of transfer task for the right and left hands using the conventional instrument.

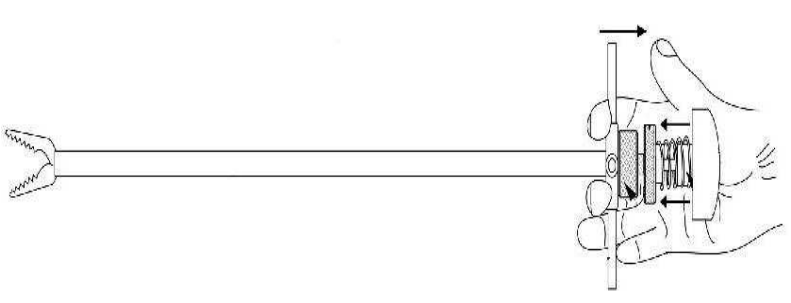

Figure 1. Ergonomic system normally closed

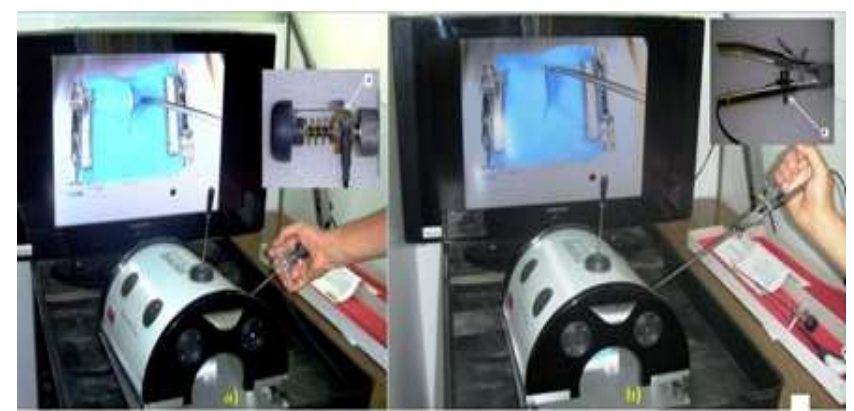

a)

b)

Figure 2 a and b. Electronic system associated to laparoscopic instruments

Table 1. Mean timing scores for the two surgeons in the transfer task using the conventional instruments.

\begin{tabular}{cccc}
\hline \multicolumn{4}{c}{ Conventional Instruments } \\
\hline \# Task & Total time (s) & Opening time (s) & Closure time (s) \\
$\mathbf{1}$ & 99.906 & 40.472 & 59.434 \\
$\mathbf{2}$ & 100.345 & 40.566 & 59.779 \\
$\mathbf{3}$ & 95.109 & 40.746 & 54.363 \\
$\mathbf{4}$ & 94.110 & 42.646 & 51.464 \\
$\mathbf{5}$ & 89.562 & 36.726 & 52.836 \\
Mean: & 95.806 & 40.231 & 55.560 \\
\hline
\end{tabular}


Table 2. Mean time scores for the two surgeons using the ergonomic instrument.

\begin{tabular}{cccc}
\hline \multicolumn{4}{c}{ Ergonomic instrument, normally closed } \\
\hline \# Task & Total time (s) & Opening time (s) & Closure time (s) \\
$\mathbf{1}$ & 76.687 & 10.226 & 66.461 \\
$\mathbf{2}$ & 71.156 & 10.566 & 60.590 \\
$\mathbf{3}$ & 70.687 & 12.372 & 58.315 \\
$\mathbf{4}$ & 86.485 & 14.865 & 71.620 \\
$\mathbf{5}$ & 73.656 & 13.262 & 60.394 \\
Mean : & 75.734 & 12.258 & 63.470 \\
\hline
\end{tabular}

\subsubsection{Transfer Task Using the Ergonomic System}

The ergonomic system was designed so that its tip is normally closed. The surgeon located the rubber ring he visually, and opened the forceps to capture the ring and hold it. The instrument closed the tip when the pressure in the handle is released. The rubber ring was secured with no stress in the surgeon hands. Then, it was transferred to the other hand. When the tips of the instruments are the middle of the visual field, the opening mechanism was activated by pressing the handle. This action allowed for transfer of the ring from one hand to the other, with the ring being secured by releasing the pressure on the handle. The surgeon moved the instrument, opens the tool tip, and deposits the ring at its final location. Table 2 shows the timing scores to complete the task using the ergonomic instrument.

Fig. 4 shows the number of openings and closure of the instrument tip in each hand, during transfer task using the new ergonomic instrument.

\subsection{Suturing Task Description}

The suturing task consists of completing a simple suture, $5 \mathrm{~cm}$ in length, through three pairs of premarked points in a longitudinal piece of cloth. The suture was tied in each point using an intracorporeal knot technique.

\subsubsection{Suturing Task Using Conventional Instrument}

The surgeon visually located the needle on the monitor. He opened the needle driver and secured the needle. At this point, the surgeon kept pressure on the instrument handle to secure the needle. He then located the first pair of points and inserted the needle in the cloth. With graspers in the other hand, he held the needle by pressing the handle, then he pulls the needle out. Once the first suture was completed, the thread was tied into a knot. This operation is repeated to complete the second and third knots. Fig. 5 shows the number of openings and closures and how long the tip is kept open or closed. Table 3 shows the mean time to complete the suture task using a conventional needle driver in the dominant hand.
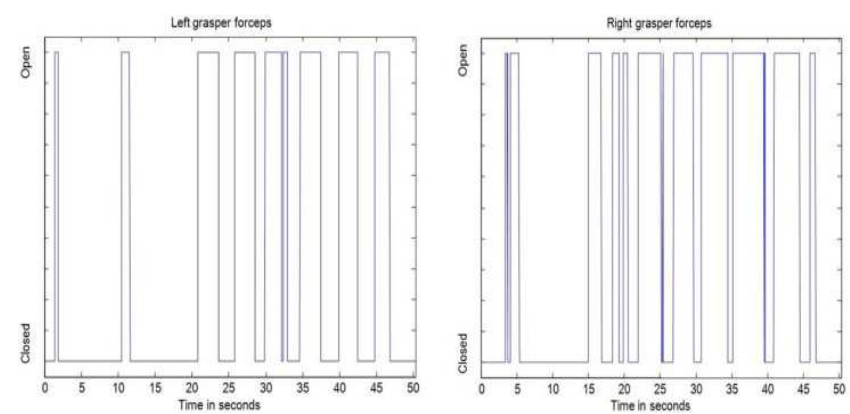

Figure 3. Opening and closure of forceps, during the third repetition of the transfer task from right to left hand using conventional instrument.
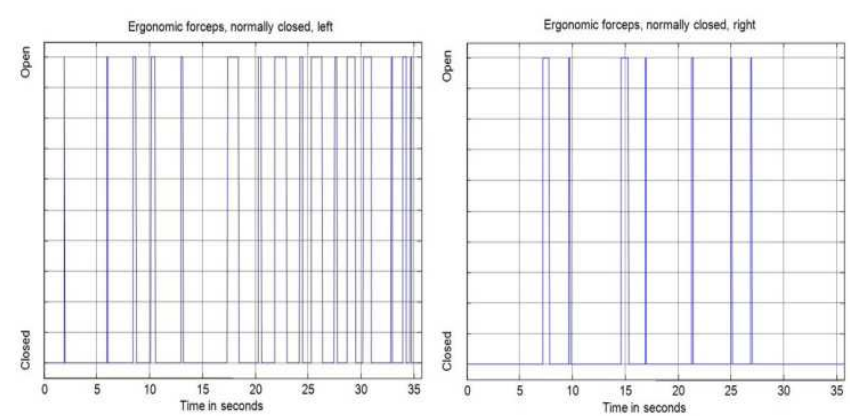

Figure 4. Opening and closure of instrument tip in each hand, during second repetition of transferring rings from right to left hand using the new ergonomic instrument

Table 3. Mean time for both surgeons to complete the suturing task, using a conventional needle driver in the dominant hand.

\begin{tabular}{ccc}
\hline Total time (s) & Opening time (s) & Closure time (s) \\
\hline 374.7500 & 177.4023 & 197.3477 \\
$100 \%$ & $47.34 \%$ & $52.66 \%$ \\
\hline
\end{tabular}

\subsubsection{Suturing Task Using the Ergonomic Instrument}

The surgeon visually located the needle, applied pressure on the handle to open the tool tip, and then released the handle. The needle was held securely in the holder tip automatically. If necessary, the final position of the needle in the driver was adjusted with the grasper tool. With the needle secured, the first point was located, and the needle was passed through the cloth. The needle driver was then released. On the other side of the cloth, the needle was secured in order to pull it and hold it at the same time. Since the needle driver was usually closed, this operation was carried out automatically.

For the next point, the same operation was performed. The surgeon then executed the second and third knots assisted with the grasper tool. Table 4 shows the mean time to complete the suture task using an ergonomic needle driver in the dominant hand. The number of openings corresponds to the number of times the surgeon opened the tool tip and how long the manual opening lasted. The number of closures corresponds to the number of times that the surgeon left the needle driver closed (Fig. 6). 
Table 4. Mean time of both surgeons to complete the suture task, using an ergonomic needle holder in the dominant hand.

\begin{tabular}{ccc}
\hline Total time (s) & Opening time (s) & Closure time (s) \\
\hline $\mathbf{3 0 0}$ & 33.9819 & 266.0181 \\
$\mathbf{1 0 0} \%$ & $11.33 \%$ & $88.67 \%$ \\
\hline
\end{tabular}

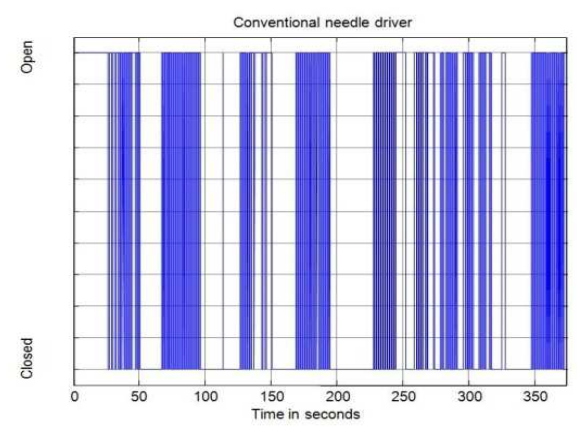

Figure 5. Openings and closures counts and how long the tip was kept closed or open using a conventional needle driver.

\section{Discussion}

The laparoscopic surgical procedure requires the surgeon to have a high degree of specialization, as well as visuospatial coordination and very fine motor coordination. The latter can be temporarily altered due to the extreme positions which the surgeon moves his extremities as a result of the positions required during surgery and the time the surgeon stays in such positions. For this reason, it is important that the surgeon has ergonomic systems that do not put him into extreme positions. Additionally, the system should be capable of reducing the time needed to complete a task. The data presented here preliminarily demonstrate that, for the ergonomic system, the time to complete the transfer task is reduced by $20 \%$ when compared to the time needed when using an instrument with a conventional handle. This reduction in time corresponds to the reduced effort the surgeon dedicates to opening the instrument. We believe the $20 \%$ reduction corresponds to the fact that the surgeon is focused on the surgical target, rather than the motor distraction of keeping the instrument tip open or closed during the task. There is an additional benefit corresponding to the closure of the instrument. Although the time of the task is not diminished, the fact that the tip is normally closed allowed a motor function reduction of $84 \%$ of the total task time.

Data show an $88 \%$ motor stress reduction using the ergonomic system during the suture task. This reduction corresponds to keeping the needle driver closed during the entire task. As a result, the task time is reduced by $20 \%$ in comparison to the conventional needle driver instrument. We believe this new surgical ergonomic system may be expanded for use in normal surgical procedures. It will not only allow for a decrease in task time, but more importantly, it will reduce the motor stress on the surgeon by decreasing the time needed and prolonged use of undesirable joint positions (elbow-wrist-fingers) during surgical procedures.

\section{Conclusions}

A new ergonomic system for handling surgical laparoscopic instruments was developed. Data indicated that, with the use of this new ergonomic instrument in the transfer and suture tasks, the number of manual operations involved in handling the instrument was reduced by $80 \%$. This was reflected in a reduction in the motor stress of the surgeon. Similarly, the results indicated that it is possible to reduce the total time needed to complete the task by $20 \%$. We are confident that we will see advantages in practice in the short-term in routine surgical procedures.

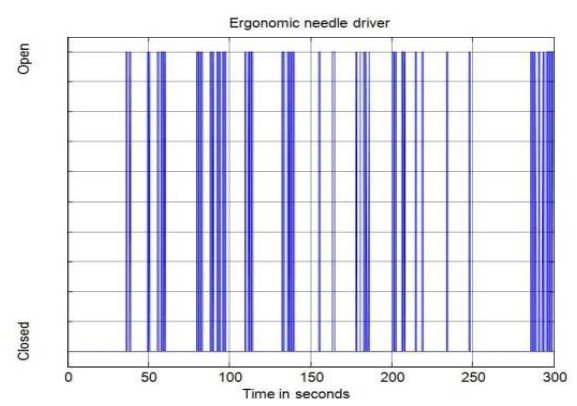

Figure 6. Openings and closures counts and how long the tip was kept closed or open using the ergonomic tool.

\section{References}

[1] D.C. Van der Zee, and N.M.A. Bax, "Digital nerve compression due to laparoscopic surgery," Surg. Endosc. vol. 9, pp. 740, 1995.

[2] U. Matern, P. Waller, "Instruments for minimally invasive surgery,” Surg. Endosc. vol. 13, pp. 174-182, 1999.

[3] U. Matern, M. Eichenlaub, P. Waller, and K.D. Rückauer, "An experimental comparison of various ergonomic handles and their design," Surg. Endosc. vol. 13, pp. 756-762, 1999.

[4] U. Matern, C. Giebmeyer, R. Bergmann, P. Waller, and M. Faist, "Ergonomic aspects of four different types of laparoscopic instrument handle with respect to elbow angle," Surg. Endosc. vol. 16, pp. 1528-1532, 2002.

[5] U. Matern, G. Kuttler, C. Giebmeyer, P. Waller, and M. Faist, "Ergonomic aspects of five different types of laparoscopic instrument handles under dynamic conditions with respect to specific laparoscopic tasks: An electromyographic-based study,” Surg. Endosc. vol. 18, pp. 1231-1241, 2004.

[6] U. Matern, S. Koneczny, M. Tedeus, K. Dietz, and G. Bue, "Ergonomic testing of two different types of handles via virtual reality simulation," Surg. Endosc. vol. 19, pp. 1147$1150,2005$.

[7] D. Büchel, R. Marvik, B. Hallabrin, and U. Matern, "Ergonomics of disposable handles for minimally invasive surgery," Surg. Endosc. vol. 24, pp. 992-1004, 2005. 